Cahiers québécois de démographie

\title{
Influence de la surmortalité masculine sur la structure par
}

sexe

Le cas italien, 1951-1981

THE IMPACT OF EXCESS MALE MORTALITY ON THE SEX

STRUCTURE

THE ITALIAN CASE, 1951-1981

INFLUENCIA DE LA SOBRE MORTALIDAD MASCULINA SOBRE

LA ESTRUCTURA POR SEXO

EL CASO ITALIANO, 1951-1981

Antonio Golini, Paolo Golini et Roberto Lombardi

Volume 18, numéro 1, printemps 1989

Démographie et femmes

URI : https://id.erudit.org/iderudit/010008ar

DOI : https://doi.org/10.7202/010008ar

Aller au sommaire du numéro

Éditeur(s)

Association des démographes du Québec

ISSN

0380-1721 (imprimé)

1705-1495 (numérique)

Découvrir la revue

Citer cette note

Golini, A., Golini, P. \& Lombardi, R. (1989). Influence de la surmortalité masculine sur la structure par sexe : le cas italien, 1951-1981. Cahiers québécois de démographie, 18(1), 195-210. https://doi.org/10.7202/010008ar
Résumé de l'article

L'analyse des relations entre l'évolution de la surmortalité masculine et celle du rapport de masculinité au sein de la population stationnaire permet de mettre en lumière le phénomène apparemment paradoxal où, pendant qu'augmente la surmortalité masculine, croît également le rapport de masculinité, du moins dans la première moitié de la vie. Ce paradoxe montre que l'indice traditionnellement adopté pour mesurer le désavantage des hommes en matière de survie, n'est pas le plus approprié.
Tous droits réservés @ Association des démographes du Québec, 1989

Ce document est protégé par la loi sur le droit d'auteur. L'utilisation des services d'Érudit (y compris la reproduction) est assujettie à sa politique d'utilisation que vous pouvez consulter en ligne.

https://apropos.erudit.org/fr/usagers/politique-dutilisation/ 
Cahiers québécois de démographie

Vol. 18, no 1, printemps 1989

\title{
Influence de la surmortalité masculine sur la structure par sexe. Le cas italien, 1951-1981
}

\author{
Antonio GOLINI*, Paolo GOLINI** \\ et Roberto LOMBARDI**
}

\section{INTRODUCTION}

L'objectif de cette note est d'analyser la surmortalité masculine en relation avec l'évolution générale de la mortalité dans un pays à faible mortalité comme l'Italie, et l'influence de cette surmortalité sur la répartition de la population par sexe.

Les variations de la mortalité en général et de la surmortalité masculine en particulier, ont un impact évident sur le rapport de masculinité au sein d'une population. L'analyse des relations entre l'évolution de la surmortalité masculine et l'évolution du rapport de masculinité au sein de la population stationnaire permet de mettre en lumière le phénomène apparemment paradoxal où, pendant que la surmortalité masculine augmente, crôt également le rapport de masculinité, du moins dans la première partie de la vie.

Ce paradoxe démontre que l'indice traditionnellement adopté pour mesurer le désavantage des hommes en matière de survie, n'est pas le plus approprié. Il est donc important de mettre au point un nouvel indice de mesure de la surmortalité masculine.

* Professeur de démographie, Département de démographie, Université "La Sapienza", Rome, et directeur de l'Istituto di Ricerche sulla Popolazione (Consiglio Nazionale delle Ricerche), Rome.

** Faculté d'économie et commerce, Université "La Sapienza", Rome. 


\section{1. ÉVOLUTION DE LA SURMORTALITÉ MASCULINE}

Aux fins de notre analyse, nous avons considéré l'évolution de la mortalité en Italie, depuis le début des années 1950 jusqu'au commencement des années 1980. Durant ces trois décennies, on a pu observer une augmentation notable de l'espérance de vie pour chacun des deux sexes, en même temps qu'une croissance significative de la surmortalité masculine.

Les chiffres du tableau 1 montrent à quel point l'évolution de l'espérance de vie a été différente pour les hommes et pour les femmes en Italie. Entre 1951 et 1981, l'espérance de vie à la naissance (c'est-à-dire la vie moyenne selon la table de mortalité) a augmenté de 7,2 années pour les hommes (une croissance de $11,4 \%$ ), mais elle a crû de 10,5 années pour les femmes (un gain de $15,6 \%$ ). Il en est résulté que l'avantage des femmes sur les hommes, en termes d'espérance de vie à la naissance, est passé de $5,5 \%$ en 1951 à 9,6\% en 1981.

Cette surmortalité masculine se vérifie à tous les âges et elle se manifeste dans tous les pays occidentaux. Elle est relativement récente. Encore au début de ce siècle, la probabilité de décès était, à certains âges, inférieure chez les hommes.

La surmortalité masculine à un âge $x$ est traditionnellement mesurée en calculant le rapport entre les probabilités masculine et féminine de décéder à cet âge. Si ce rapport est supérieur à l'unité, il y a effectivement surmortalité masculine; la différence entre ce rapport et l'unité indique de combien (en pourcentage) la mortalité masculine est supérieure à la mortalité féminine. Le tableau 2 présente le résultat de ces calculs, selon les conditions de mortalité observées en Italie en 1950-1953, 1970-1972 et 1979-1983. 


\section{Tableau 1}

Espérance de vie à la naissance.

Italie, 1950-1953 à 1979-1983

Espérance de vie

1950-1953

1970-1972

1979-1983

Variation absolue

1951-1971

1971-1981

1951-1981

Hommes (H) Femmes (F) Rapport H/F

$\begin{array}{lll}63,71 & 67,24 & 1,055 \\ 68,97 & 74,88 & 1,086 \\ 70,95 & 97,73 & 1,096\end{array}$

Variation relative (en \%)

1951-1971

1971-1981

1951-1981

$\begin{array}{rrr}5,26 & 7,64 & 0,031 \\ 1,98 & 2,85 & 0,010 \\ 7,24 & 10,49 & 0,041\end{array}$

Source : Institut national de statistique (ISTAT, 1957, 1975, 1987)

L'évolution selon l'âge est relativement semblable au cours du temps, avec deux sommets, l'un à 20 ans (accidents de la route) et l'autre entre 50 et 60 ans (tumeurs et maladies cardio-vasculaires). Alors qu'en 1950-1953 ce second sommet était encore nettement supérieur au premier, tel n'est plus le cas en 1970-1972 et 1979-1983. 
Tableau 2

Évolution de la surmortalité masculine, selon l'âge.

Italie, 1950-1953 aे 1979-1983

\begin{tabular}{rccc}
\hline Age & $1950-1953$ & $1970-1972$ & $1979-1983$ \\
\hline 0 & 1,148 & 1,244 & 1,271 \\
5 & 1,137 & 1,318 & 1,333 \\
10 & 1,344 & 1,593 & 1,611 \\
15 & 1,355 & 2,343 & 2,481 \\
20 & 1,413 & 2,510 & 3,028 \\
25 & 1,321 & 2,036 & 2,676 \\
30 & 1,234 & 1,809 & 2,085 \\
35 & 1,246 & 1,660 & 1,803 \\
40 & 1,293 & 1,799 & 1,858 \\
45 & 1,505 & 1,948 & 2,011 \\
50 & 1,654 & 1,876 & 2,216 \\
55 & 1,635 & 1,990 & 2,371 \\
60 & 1,531 & 2,021 & 2,336 \\
65 & 1,310 & 1,975 & 2,202 \\
70 & 1,199 & 1,756 & 2,009 \\
75 & 1,152 & 1,493 & 1,762 \\
80 & 1,123 & 1,258 & 1,519 \\
85 & 1,124 & 1,187 & 1,360 \\
90 & 1,141 & 1,210 & 1,228 \\
95 & 1,159 & 1,230 & 1,121 \\
100 & 1,168 & 1,232 & 1,038 \\
\hline
\end{tabular}

Note : La surmortalité masculine à un âge $\mathrm{x}$ est obtenue en calculant le rapport entre les probabilités masculine et féminine de décéder à cet âge.

D'une période à l'autre, la surmortalité masculine a augmenté à tous les âges, mais l'augmentation a été particulièrement forte entre 15 et 30 ans, à tel point qu'en 1979-1983, la probabilité masculine de décéder à 20 ans était trois fois plus forte que la probabilité féminine, alors que la surmortalité masculine à cet âge n'était que de $40 \%$ en 1950-1953. Selon les conditions de mortalité observées en 1979-1983, la probabilité masculine de décéder est toujours au moins le double de la probabilité féminine pour tous les âges entre 45 et 70 ans, alors qu'en 1950-1953, à ces mêmes âges, la surmortalité masculine n'était "que" de $20 \%$ (à 70 ans) à $65 \%$ (à 50 ans). Une telle évolution est évidemment à mettre en relation avec le fait que certaines causes de décès (maladies cardio-vasculaires, accidents et autres causes violentes), vis-à-vis 
desquelles les femmes semblent mieux protégées que les hommes, ont pris une importance relative de plus en plus considérablel.

\section{2. ÉVOLUTION DU RAPPORT DE MASCULINITÉ DANS LA POPU- LATION STATIONNAIRE}

Le rapport de masculinité dans une population à un âge $\mathrm{x}$ est obtenu en divisant l'effectif de la population masculine de cet âge à l'effectif de la population féminine ayant le même âge. Si, à la naissance, ce rapport est généralement supérieur à l'unité, il diminue continuellement par la suite, en relation avec la surmortalité masculine observée à tous les âges (du moins en Italie). Dans le cadre de notre analyse, nous examinerons l'évolution de ce rapport dans la population stationnaire telle qu'exprimée à partir des tables italiennes de mortalité de 1950-1953, 1970-1972, et 1979-1983. Ceci nous permettra de ne prendre en compte que la seule surmortalité masculine "normale", c'est-à-dire excluant celle due par exemple à la guerre. II nous faut cependant supposer que la population italienne est close, c'est-à-dire non soumise à migration.

Comme le montrent les chiffres du tableau 3, l'avantage numérique que les hommes ont sur les femmes au moment de la naissance diminue avec l'âge, mais subsiste jusqu'à environ 53 ans. Quelle que soit la période envisagée, c'est toujours entre 50 et 55 ans que le rapport de masculinité (dans la population stationnaire) descend en dessous de l'unité. Mais après 55 ans, le rapport baisse rapidement, de telle sorte qu'à 85 ans il n'y a plus que 48 hommes pour 100 femmes selon le régime de mortalité de 1979-1983. Le rapport était encore de 73 hommes pour 100 femmes en 1950-1953.

1. Selon un rapport récent des Nations Unies, portant sur la contribution des diverses causes de décès à la différence des espérances de vie entre hommes et femmes, pour un ensemble de 26 pays développés au début des années 1980 , près de $40 \%$ de la différence serait due aux maladies cardiovasculaires et près de $20 \%$ aux accidents et autres causes violentes, les tumeurs contribuant pour $18 \%$ (United Nations, 1987; voir également Waldron, 1985). 
La forte augmentation de la surmortalité masculine entre 1950-1953 et 1979-1983 se reflète en fait essentiellement sur le rapport de masculinité après 50 ans. Jusqu'à cet âge, tous les rapports de masculinité de 1979-1983 sont supérieurs à ceux de 1950-1953 (cela n'est cependant pas vrai pour les rapports de masculinité de 1970-1973, qui sont inférieurs à ceux de 1950-1953 à partir de 30 ans).

Tableau 3

Évolution du rapport de masculinité dans la population stationnaire, selon l'âge. Italie, 1950-1953 à 1979-1983

\begin{tabular}{rccc}
\hline Age & $1950-1953$ & $1970-1972$ & $1979-1983$ \\
\hline 0 & 105,50 & 105,50 & 105,50 \\
5 & 104,47 & 104,79 & 105,13 \\
10 & 104,39 & 106,71 & 105,08 \\
15 & 104,29 & 104,59 & 104,90 \\
20 & 104,09 & 104,26 & 104,68 \\
25 & 103,85 & 103,92 & 104,32 \\
30 & 103,66 & 103,63 & 104,02 \\
35 & 103,43 & 103,33 & 103,74 \\
40 & 103,14 & 102,90 & 103,38 \\
45 & 102,53 & 102,09 & 102,73 \\
50 & 101,29 & 100,77 & 101,49 \\
55 & 99,25 & 98,66 & 99,14 \\
60 & 96,36 & 95,29 & 95,29 \\
70 & 93,01 & 90,08 & 89,81 \\
75 & 85,70 & 74,20 & 72,15 \\
80 & 80,62 & 65,67 & 60,27 \\
85 & 72,92 & 57,83 & 68,00 \\
90 & 61,03 & 47,35 & 36,59 \\
95 & 44,30 & 33,02 & 27,36 \\
100 & 26,40 & 18,60 & 21,54 \\
\hline
\end{tabular}

Note : Le rapport de masculinité à l'âge $\mathrm{x}$ est obtenu en divisant l'effectif de la population (stationnaire) masculine de cet âge à l'effectif correspondant de la population féminine. 


\section{RELATION ENTRE ÉVOLUTION DE LA SURMORTALITÉ MAS- CULINE ET ÉVOLUTION DU RAPPORT DE MASCULINITÉ}

Nous venons de voir que, malgré la forte augmentation de la surmortalité masculine au cours des trois dernières décennies, le nombre d'hommes survivants (dans la population stationnaire) a augmenté plus que proportionnellement à celui des femmes, du moins pour les cinquante premières années de la vie. $\mathrm{Ce}$ paradoxe implique que l'indice traditionnellement utilisé pour mesurer la surmortalité masculinité n'est pas nécessairement le plus approprié si l'on veut évaluer l'impact sur la structure par sexe d'une population (Golini, 1967 et Keyfitz et Golini, 1975).

On peut en effet démontrer que le rapport de masculinité est une fonction de la surmortalité masculine, à condition que cette surmortalité soit mesurée non par le rapport entre les probabilités de décès (masculine et féminine, respectivement), mais par la différence entre ces probabilités.

Pour illustrer notre propos, nous prendrons l'exemple théorique au tableau 4.

Dans une première situation, celle du cas $\mathrm{A}$, la chute de la mortalité féminine (de $80 \mathrm{o} / 00$ à $20 \%$ oo, soit une baisse de $60 \mathrm{o} / 00$ ) est inférieure en termes absolus à celle de la mortalité masculine (de $100 \mathrm{o} / 00$ à $30 \%$ oo, soit une baisse de $70 \mathrm{o} / 00$ ) tout en étant supérieure en termes relatifs (ce qui permet à la surmortalité masculine, mesurée par le rapport des probabilités, d'augmenter). Dans ce cas, à l'âge d'un an les hommes compteront plus de survivants supplémentaires (par rapport au moment initial, celui qui précède la baisse de la mortalité) que les femmes (le gain étant dans notre exemple de 73 pour les hommes et de 60 pour les femmes), ce qui, combiné au fait qu'il nât plus d'hommes que de femmes, permet à l'écart entre le nombre de survivants à l'âge d'un an de passer de 30 à 43 en faveur des hommes. Corrélativement, le rapport de masculinité augmente (en l'occurrence, de 1,033 à 1,044). 
Tableau 4

Relation entre surmortalité masculine et rapport de masculinité.

Cas théoriques

\begin{tabular}{lccc}
\hline & Temps t & \multicolumn{2}{c}{ Temps t+1 } \\
& & Cas A & Cas B \\
$\begin{array}{l}\text { (1) Probabilités de décès (o/oo) } \\
\text { Hommes }\end{array}$ & 100 & 30 & 45 \\
$\begin{array}{l}\text { (2) Probabilités de décès (o/oo) } \\
\text { Femmes }\end{array}$ & 80 & 20 & 20 \\
$\begin{array}{l}\text { (3) Rapport des probabilités : } \\
\text { (1)/ (2) }\end{array}$ & 1,25 & 1,50 & 2,25 \\
$\begin{array}{l}\text { (4) Différence des probabilités : } \\
\text { (1) - (2) }\end{array}$ & 20 & 10 & 25 \\
$\begin{array}{l}\text { (5) Nombre d'hommes survivant } \\
\text { à l'âge d'un ana }\end{array}$ & 950 & 1023 & 1008 \\
$\begin{array}{l}\text { (6) Nombre de femmes survivant } \\
\text { à l'âge d'un anà }\end{array}$ & 920 & 980 & 980 \\
$\begin{array}{l}\text { (7) Rapport de masculinité à } \\
\text { l'âge 1 }\end{array}$ & 1,033 & 1,044 & 1,029 \\
\hline
\end{tabular}

a. En supposant (comme cela est le cas en Italie et dans la plupart des pays) qu'il y a 1055 naissances masculines pour 1000 naissances féminines.

Par contre, si l'augmentation de la surmortalité masculine est plus que proportionnelle à la chute de la mortalité, c'est-à-dire si la différence (en termes absolus) entre les probabilités masculine et féminine de décéder a augmenté au cours de la période, comme dans le cas B du tableau 4, alors le gain (en termes de nombre de survivants) sera inférieur pour les hommes au gain réalisé par les femmes (dans notre exemple, il y aurait 58 survivants de plus parmi les 1055 naissances masculines, et 60 survivantes de plus parmi les 1000 naissances féminines). Il en résulte nécessairement une baisse du rapport de masculinité. 
On peut formaliser de la façon suivante la relation entre rapport de masculinité et surmortalité :

$$
\begin{aligned}
& P x^{h} / P^{f}=\left(N^{h} / N^{f}\right) .(\text { Sox } h / S o x f) \\
& =\frac{\text { lo. }(1.055) \cdot \text { Sox }^{h}}{10 \cdot \text { Sox }^{f}} \\
& =\frac{1.055\left(\operatorname{Sox} f^{f}-D 0 x\right)}{S_{0 x}^{f}} \\
& =1,055\left(1-\operatorname{Dox} / \operatorname{Sox}^{f}\right) \text {, où }
\end{aligned}
$$

$\mathrm{Px}^{\mathrm{h}}$ et $\mathrm{Px}^{\mathrm{f}}$ representent le nombre de survivants à l'âge $\mathrm{x}$, pour les hommes et les femmes respectivement;

$\mathrm{N}^{\mathrm{h}}$ et $\mathrm{N}^{\mathrm{f}}$ indiquent le nombre de naissances, masculines et féminines respectivement;

Sox ${ }^{\mathrm{h}}$ et Sox ${ }^{\mathrm{f}}$ sont les probabilités de survie, depuis la naissance jusqu'à l'âge $\mathrm{x}$, pour les hommes et les femmes respectivement;

lo est la racine de la table de mortalité, c'est-à-dire l'effectif initial de la cohorte de la table (généralement, une puissance de 10); et où

Dox représente la différence entre les probabilités de décès féminine et masculine, c'est-à-dire Dox $=\operatorname{Sox}^{\mathrm{f}}-\operatorname{Sox}^{\mathrm{h}}$.

L'équation (1) permet de mettre en évidence le rôle joué par la différence entre les probabilités de survie, par définition égale à la différence entre les probabilités de décès, dans la détermination du rapport de masculinité d'une population. 


\section{LA FONCTION "DIFFÉRENCE" COMME MESURE DE LA SUR- MORTALITÉ MASCULINE}

Nous venons de voir, grâce à l'équation (1), qu'à une constante près, le rapport de masculinité d'une population d'âge $\mathrm{x}$ est directement fonction du rapport entre la différence des probabilités masculine et féminine de survivre de la naissance jusqu'à l'âge $\mathrm{x}$ (ou des probabilités de décéder avant l'âge $\mathrm{x}$ ). Puisque ce dernier rapport est nécessairement compris entre 0 et 1 , et qu'il est soustrait de l'unité, il s'ensuit que le rapport de masculinité à un âge quelconque $\mathrm{x}$ est nécessairement inférieur au rapport de masculinité à la naissance $(1,055$ en l'occurrence). En outre, puisque l'écart entre les probabilités de survie augmente en général avec l'âge, du moins si l'on exclut les âges avancés (voir ci-dessous), il résulte également de l'équation (1) que le rapport de masculinité d'une population d'âge $x$ décroît normalement lorsque l'âge augmente.

Le tableau 5 présente l'évolution (de 1950-1953 à 1979-1983) de l'écart (Dox) entre les probabilités (en o/oo) masculine et féminine de survivre jusqu'à chaque âge $x$, pour la population italienne. On remarquera qu'en 1979-1983, l'écart demeure inférieur à celui observé en 1950-1953 pour tous les groupes d'âge inférieurs à $45-49$ ans, ce qui se reflétait dans le fait que le rapport de masculinité a augmenté pendant la même période pour tous les groupes d'âge jusqu'à 50-54 ans (voir tableau 3).

Cet indicateur Dox, qui représente donc la différence entre la courbe des survivantes et la courbe des survivants à l'âge $\mathrm{x}$ (et par conséquent, le nombre d'années de vie perdues par la population masculine par rapport à la population féminine ${ }^{2}$ ), semble être une bonne mesure globale de la surmortalité masculine depuis la naissance jusqu'à l'âge $\mathrm{x}$. Les résultats du tableau 5 montrent qu'en Italie l'écart maximum observé en 1950-1953 était à l'âge de 72 ans, avec une valeur de 96 o/oo, et que cet écart se présente à des âges de plus en plus

2. Dans un article récent, Maccheroni (1988) adopte - de façon tout à fait indépendante - cette approche. 
avancés, avec des valeurs de plus en plus élevées : en 1970-1972 il était monté à 192 o/oo à l'âge de 76 ans, et en fin de période, en 1979-1983, il s'élevait à 233 o/oo à l'âge de 78 ans.

Tableau 5

Évolution de la différence entre les probabilités (en o/oo) masculine et féminine de survivre depuis la naissance jusqu'à l'âge x. Italie, 1950-1953 à 1979-1983

\begin{tabular}{|c|c|c|c|}
\hline Age & $1950-1953$ & $1970-1972$ & $1979-1983$ \\
\hline 0 & 0,00 & 0,00 & 0,00 \\
\hline 5 & 9,00 & 6,55 & 3,49 \\
\hline 10 & 9,69 & 7,27 & 3,88 \\
\hline 15 & 10,57 & 8,39 & 4,59 \\
\hline 20 & 12,21 & 11,37 & 7,64 \\
\hline 25 & 14,21 & 14,49 & 10,98 \\
\hline 30 & 15,95 & 17,07 & 13,72 \\
\hline 35 & 17,56 & 19,71 & 16,28 \\
\hline 40 & 19,79 & 23,49 & 19,52 \\
\hline 45 & 24,52 & 30,67 & 25,32 \\
\hline 50 & 34,06 & 41,72 & 36,27 \\
\hline 55 & 49,03 & 58,76 & 56,57 \\
\hline 60 & 68,26 & 85,06 & 88,24 \\
\hline 65 & 86,10 & 121,57 & 129,40 \\
\hline 68 & 95,17 & 162,33 & 158,06 \\
\hline 69 & 94,92 & 156,59 & 167,98 \\
\hline 70 & 95,17 & 162,33 & 177,81 \\
\hline 71 & 95,64 & 169,61 & 187,50 \\
\hline 72 & 95,83 & 176,27 & 196,95 \\
\hline 73 & 95,33 & 182,09 & 205,90 \\
\hline 74 & 93,91 & 186,78 & 214,13 \\
\hline 75 & 91,93 & 190,02 & 221,29 \\
\hline 76 & 89,77 & 191,58 & 227,03 \\
\hline 77 & 87,12 & 191,20 & 231,11 \\
\hline 78 & 83,50 & 188,68 & 233,07 \\
\hline 79 & 78,76 & 184,02 & 232,68 \\
\hline 80 & 73,69 & 177,28 & 229,88 \\
\hline 81 & 68,39 & 168,68 & 224,74 \\
\hline 82 & 62,93 & 158,35 & 217,63 \\
\hline 85 & 45,30 & 121,39 & 182,01 \\
\hline 90 & 18,96 & 59,58 & 96,78 \\
\hline 95 & 4,67 & 18,94 & 27,87 \\
\hline
\end{tabular}

Si, au lieu d'une mesure globale de la surmortalité (par exemple, depuis la naissance jusqu'à l'âge $\mathrm{x}$ ), on désire une mesure ponctuelle de la 
surmortalité à un âge déterminé, on aura intérêt à prendre la différence entre les probabilités de décès spécifiques à cet âge. Le tableau 6 présente l'évolution de cet écart à chaque âge. En comparant cette évolution de la surmortalité mesurée en termes de différence (entre probabilités masculine et féminine de décéder à un âge donné) à l'évolution mesurée en termes de rapport des probabilités (comme cela se fait habituellement), on peut mettre en lumière certains phénomènes intéressants.

On peut voir ainsi (deux premières lignes du tableau 6), que même si pour les âges jeunes ( 0 à 14 ans) la surmortalité masculine, mesurée par le rapport des probabilités de décès, a augmenté entre 1950-1953 et 1979-1983 (c'est-à-dire que la probabilité de décès à ces âges a moins baissé pour les hommes que pour les femmes), cela n'a eu aucune influence négative sur le rapport de masculinité, au contraire. En réalité, toutes autres choses étant égales, cela a eu pour effet d'augmenter le rapport de masculinité, puisque l'écart entre les probabilités a diminué considérablement (l'écart étant réduit de plus de la moitié à 0-4 ans, et de près de la moitié entre 5 et 14 ans). Or, comme nous l'avons montré par l'équation (1), une baisse de l'écart des probabilités (même s'il y a eu hausse dans le rapport de ces mêmes probabilités) entraîne une augmentation du rapport de masculinité. Cet effet à la hausse est cependant tempéré par le fait que, dans l'équation (1), cet écart plus faible est pondéré par la probabilité de survie des femmes, probabilité qui, elle, a augmenté.

Par contre, aux âges avancés (après 65 ans), la surmortalité mesurée par différence manifeste une détérioration (pour les hommes par rapport aux femmes) beaucoup plus marquée que celle exprimée par le rapport des probabilités, et cette détérioration maximale ne s'observe pas aux mêmes âges : si l'on considère la surmortalité par le rapport des probabilités, cette surmortalité a augmenté le plus entre 65 et 74 ans (avec une hausse de $68 \%$ du rapport, entre 1950-1953 et 1979-1983), alors que si l'on mesure la surmortalité par la différence des probabilités, c'est entre 75 et 79 ans que la hausse est la 
Tableau 6

Évolution de la différence entre les probabilités (en o/oo) masculine et féminine de décéder à un âge $\mathrm{x}$ donné $(\mathrm{qx})$.

Italie, 1950-1953 à 1979-1983

\begin{tabular}{|c|c|c|c|c|c|c|}
\hline \multirow[b]{2}{*}{ Age } & \multicolumn{3}{|c|}{$D x=q x^{h}-q x^{f}$} & \multicolumn{3}{|c|}{$\begin{array}{c}\text { Variation } \\
1950-53 \text { à } 1979-83\end{array}$} \\
\hline & $1950-53$ & $1970-72$ & $1979-83$ & $D x$ & $\% \mathrm{Dx}$ & $\% \mathrm{qx}^{\mathrm{h}} / \mathrm{qx}$ \\
\hline 0 & 8,78 & 6,05 & 3,26 & $-5,52$ & $-62,9$ & 10,7 \\
\hline 5 & 0,15 & 0,14 & 0,08 & $-0,07$ & $-46,7$ & 17,2 \\
\hline 10 & 0,20 & 0,16 & 0,11 & $-0,09$ & $-45,0$ & 19,9 \\
\hline 15 & 0,27 & 0,47 & 0,40 & 0,13 & 48,1 & 83,1 \\
\hline 20 & 0,45 & 0,71 & 0,71 & 0,26 & 57,8 & 114,3 \\
\hline 25 & 0,56 & 0,58 & 0,62 & 0,06 & 10,7 & 102,6 \\
\hline 30 & 0,38 & 0,55 & 0,51 & 0,13 & 34,2 & 68,9 \\
\hline 35 & 0,49 & 0,66 & 0,57 & 0,08 & 16,3 & 44,7 \\
\hline 40 & 0,76 & 1,19 & 0,97 & 0,21 & 27,6 & 43,7 \\
\hline 45 & 1,85 & 2,19 & 1,84 & $-0,01$ & $-0,0$ & 33,6 \\
\hline 50 & 3,44 & 3,33 & 3,60 & 0,16 & 4,7 & 34,0 \\
\hline 55 & 5,08 & 5,66 & 6,43 & 1,35 & 26,6 & 45,0 \\
\hline 60 & 6,71 & 9,08 & 9,98 & 3,27 & 48,7 & 52,6 \\
\hline 65 & 6,82 & 14,16 & 14,60 & 7,78 & 114,1 & 68,1 \\
\hline 70 & 7,72 & 19,07 & 21,43 & 13,71 & 177,6 & 67,6 \\
\hline 75 & 10,43 & 23,64 & 30,42 & 19,99 & 191,7 & 52,9 \\
\hline 80 & 14,15 & 21,72 & 37,63 & 23,48 & 165,9 & 35,3 \\
\hline 85 & 22,33 & 26,03 & 43,80 & 21,47 & 96,1 & 21,0 \\
\hline 90 & 36,38 & 43,92 & 44,63 & 8,25 & 22,7 & 7,6 \\
\hline 95 & 54,23 & 65,42 & 37,46 & $-16,77$ & $-30,9$ & $-3,3$ \\
\hline 100 & 70,38 & 83,70 & 17,20 & $-53,18$ & $-75,6$ & $-11,1$ \\
\hline
\end{tabular}

plus forte (de près de $200 \%$ ). Dans ces conditions, il n'est guère surprenant que la baisse du rapport de masculinité se manifeste surtout après 75 ans (voir tableau 3), même si la hausse de la surmortalité masculine (mesurée par le rapport des probabilités de décès) a été beaucoup plus forte entre 15 et 34 ans et entre 65 et 74 ans, qu'après 75 ans. 


\section{CONCLUSION}

Mesurer la surmortalité masculine en termes de différence entre les probabilités de décès masculine et féminine plutôt qu'en termes de rapport des probabilités, présente l'avantage de pouvoir estimer de façon immédiate l'impact de cette surmortalité sur le rapport de masculinité au sein d'une population. De plus, l'image qu'on peut avoir de l'évolution de cette surmortalité sera plus significative, surtout si l'on compare la surmortalité à différents âges. Pour les groupes d'âge des enfants et des jeunes adultes, l'utilisation du rappport des probabilités de décès peut amener à conclure qu'il y a eu une très forte croissance de la surmortalité, alors qu'en réalité les écarts ne se sont accrus que relativement peu, et même ont diminué dans certains cas; par contre, pour les groupes d'âge avancé, les écarts peuvent s'être accrus considérablement alors que, d'après le rapport des probabilités, on aurait pu conclure que la surmortalité masculine n'a pas augmenté plus que dans la plupart des autres groupes d'âge. 


\section{RÉFÉRENCES BIBLIOGRAPHIQUES}

GOLINI, Antonio, 1967. "L'influenza delle variazioni di mortalità sulla struttura per sesso della popolazione". In Atti della XXVa Riunione scientifica delle Società italiana di statistica, Bologna.

ISTAT, 1957. "Tavole di mortalità per sesso, 1950-1953. Annuario italiano di statistica. Rome, Institut national de statistique.

ISTAT, 1975. "Tavole di mortalità della popolazione italiana, 1970-1972". Bollettino mensile di statistica, no 1, Rome, Institut national cle statistique.

ISTAT, 1987. "Tavole di mortalità della popolazione italiana per regione, 1979-1983". Note e relazioni, no 1, Rome, Institut national de statistique.

KEYFITZ, Nathan et Antonio GOLINI, 1975. "Mortality comparisons. The male-female ratio". Genus, 31, 1-4.

MACCHERONI, Carlo, 1988. "La mortalité différentielle mesurée en termes de dissemblance. Les écarts de mortalité entre sexes selon les tables italiennes du XXe siècle". Cahiers québécois de démographie, 17, 1, 6589.

UNITED NATIONS, 1987. "Are sex differences in life expectancy on the increase ?". Population Newsletter, no 44.

WALDRON, Ingrid, 1985. "Que savons-nous de la différenciation sexuelle dans la mortalité ? Aperçu bibliographique". Bulletin démographique des Nations Unies, no 18, 67-87. 


\section{RÉSUMÉ - SUMMARY - RESUMEN}

GOLINI Antonio, Paolo GOLINI et Roberto LOMBARDI - INFLUENCE DE LA SURMORTALITÉ MASCULINE SUR LA STRUCTURE PAR SEXE. LE CAS ITALIEN, 1951-1981

L'analyse des relations entre l'évolution de la surmortalité masculine et celle du rapport de masculinité au sein de la population stationnaire permet de mettre en lumière le phénomène apparemment paradoxal où, pendant qu'augmente la surmortalité masculine, croît également le rapport de masculinité, du moins dans la première moitié de la vie. Ce paradoxe montre que l'indice traditionnellement adopté pour mesurer le désavantage des hommes en matière de survie, n'est pas le plus approprié.

GOLINI, Antonio, Paolo GOLINI and Roberto LOMBARDI - THE IMPACT OF EXCESS MALE MORTALITY ON THE SEX STRUCTURE. THE ITALIAN CASE, 1951-1981

The analysis of the links between the evolution of excess male mortality and of the evolution of the sex ratio in the stationary population, allows for a better understanding of the apparently paradoxical phenomenon where at least during the first half of life, the sex ratio increases when excess male mortality increases. This paradox shows that the index traditionally used for measuring the disadvantage of men with regard to survival, is not the most appropriate one.

GOLINI Antonio, Paolo GOLINI y Roberto LOMBARDI - INFLUENCIA DE LA SOBREMORTALIDAD MASCULINA SOBRE LA ESTRUCTURA POR SEXO. EL CASO ITALIANO, 1951-1981

El análisis de las, relaciones entre la evolución de la sobremortalidad masculina y el de la relación de masculinidad en el seno de la población estacionaria permite poner a luz el fenómeno aparentemente paradójico donde, mientras aumenta la sobremortalidad masculina, crece igualmente la relación de masculinidad, por lo menos en la primera mitad de la vida. Esta paradoja muestra que el índice tradicionalmente adoptado para medir la desventaja de los hombres en materia de sobrevivencia, no es el más apropiado. 